\title{
BMJ Open Evaluating the implementation and effectiveness of a multi-component intervention to reduce post-surgical opioid prescribing: study protocol of a mixed-methods design
}

\author{
Jonah J Stulberg, ${ }^{\circledR 1,2}$ Willemijn L A Schäfer, ${ }^{1}$ Meagan L Shallcross, ${ }^{\circledR} 1$ \\ Bruce L Lambert, ${ }^{3}$ Reiping Huang, ${ }^{1}$ Jane L Holl, ${ }^{1,2}$ Karl Y Bilimoria, ${ }^{1,2}$ \\ Julie K Johnson ${ }^{1,2}$
}

To cite: Stulberg JJ, Schäfer WLA, Shallcross ML, et al. Evaluating the implementation and effectiveness of a multicomponent intervention to reduce post-surgical opioid prescribing: study protocol of a mixedmethods design. BMJ Open 2019;9:e030404. doi:10.1136/ bmjopen-2019-030404

Received 12 March 2019 Revised 16 April 2019 Accepted 7 May 2019
Check for updates

(C) Author(s) (or their employer(s)) 2019. Re-use permitted under CC BY-NC. No commercial re-use. See rights and permissions. Published by BMJ.

For numbered affiliations see end of article.

Correspondence to Dr Jonah J Stulberg; jonah.stulberg@nm.org

\begin{abstract}
Introduction Opioids prescribed after surgery accounted for $5 \%$ of the 191 million opioid prescriptions filled in 2017. Approximately $80 \%$ of the opioid pills prescribed by surgical care providers remain unused, leaving a substantial number of opioids available for non-medical use. We developed a multi-component intervention to address surgical providers' role in the overprescribing of opioids. Our study will determine effective strategies for reducing post-surgical prescribing while ensuring adequate post-surgery patient-reported pain-related outcomes, and will assess implementation of the strategies.
\end{abstract}

Methods and analysis The Minimising Opioid Prescribing in Surgery study will implement a multi-component intervention, in an Illinois network of six hospitals (one academical, two large community and three small community hospitals), to decrease opioid analgesics prescribed after surgery. The multi-component intervention involves four domains: (1) patient expectation setting, (2) baseline assessment of opioid use, (3) perioperative pain control optimisation and (4) post-surgical opioid minimisation. Four surgical specialities (general, orthopaedics, urology and gynaecology) at the six hospitals will implement the intervention. A mixed-methods approach will be used to assess the implementation and effectiveness of the intervention. Data from the network's enterprise data warehouse will be used to evaluate the intervention's effect on post-surgical prescriptions and a survey will collect pain-related patient-reported outcomes. Intervention effectiveness will be determined using a triangulation design, mixed-methods approach with staggered speciality-specific implementation for contemporaneous control of opioid prescribing changes over time. The Consolidated Framework for Implementation Research will be used to evaluate the site-specific contextual factors and adaptations to achieve implementation at each site.

Ethics and dissemination The study aims to identify the most effective hospital-type and speciality-specific intervention bundles for rapid dissemination into our 56-hospital learning collaborative and in hospitals
Strengths and limitations of this study

- Iterative modification of the evidence-based components of the intervention will allow us to study how hospitals and specialities adapt and improve the components to meet their site-specific contextual needs.

- The study will provide a generalisable framework for tailored implementation of post-surgical discharge pain management prescribing best practices to reduce patient and societal harm due to excess opioids without negatively affecting patient reported pain outcomes.

- Comparison to within site non-intervention controls allows us to control for secular decline in opioid prescriptions, driven by the salience of the 'opioid epidemic' in popular press and public policy.

- The study duration (3years) is a relatively short time to expect complete implementation and to observe improvement.

throughout the USA. All study activities have been approved by the Northwestern University Institutional Review Board (ID STU00205053).

\section{INTRODUCTION}

\section{Background and existing literature}

Nearly all surgical procedures result in some level of postoperative pain, although with varying intensity and duration, for which surgical providers prescribe pain medications, often opioids. In our prior study, in a single health system, opioids were prescribed after $95 \%$ of surgical procedures. ${ }^{1}$ Furthermore, we found that the patients only used, on average, a small number of pills $(\sim 5$ pills) after elective general surgical procedures, ${ }^{1}$ confirming prior studies' finding that between $70 \%$ to $90 \%$ of prescribed opioids 
after surgery remain unused by the patient. ${ }^{2-5}$ It has also been established that only a small fraction of unused opioid pills are properly disposed of ${ }^{6-8}$ leaving a substantial number of pills available for potential non-medical use and diversion. ${ }^{59}$

Indeed, $50 \%$ to $75 \%$ of non-medical opioid users obtain the drugs from relatives or friends, a concept known as 'diversion'. ${ }^{11}$ In 2016, 11.5 million individuals (>12 years) reported using prescription drugs for a non-medical purpose in the past year. ${ }^{12}$ Every day, more than 115 people die after overdosing on opioids and, despite recent efforts to curb the crisis, the numbers are still steadily increasing. ${ }^{13}$ Moreover, opioid related poisoning caused 78840 hospitalisations and 140077 emergency department visits in 2015 alone. ${ }^{12}$ Finally, more than $80 \%$ of people who use heroin have a history of non-medical use of pain relievers first. ${ }^{114}$ Much of the focus has fallen on opioid prescribing by primary care physicians, particularly for chronic opioid use, but surgical providers also have a role in this epidemic, given the overprescribing of opioids after surgery.

While opioid prescriptions written by surgical providers, in 2017, accounted for only 5\% of the 191 million dispensed opioid prescriptions in the USA, ${ }^{15}$ they nevertheless accounted for 10.4 million filled prescriptions. ${ }^{16}$ Many factors contribute to opioid prescribing decisions for pain control following a surgical procedure, including patient factors (eg, pain tolerance, opioid tolerance), procedural factors (eg, open incision, laparoscopic incision), legal influences (eg, laws that restrict the ability to electronically prescribe or offer refills remotely) and social factors (eg, distance to provider to obtain a refill prescription). ${ }^{3}$ 17-39 Research also shows that surgical providers often use a default number of pills (eg, 60 pills after hernia surgery) with little attention to patient factors, often prescribing more pills than needed for the majority of the patients. 35274041

The role of surgical providers in the opioid epidemic unfolds in three ways ${ }^{1}$ : (i) overprescribing of opioids following surgery, which leaves unused opioids available for non-medical use and diversion ${ }^{2-5}$, (ii) inadequately providing perioperative patient education around safe use and proper disposal of unused opioid pills and expectation setting about post-surgical pain and pain management ${ }^{174243}$ and $^{3}$ (iii) contributing to the development of chronic post-surgical pain. ${ }^{35} 174042-46$

It is imperative that the numerous factors influencing surgical provider opioid prescribing be addressed while ensuring that the patients still receive adequate post-surgical pain control. Minimising Opioid Prescribing in Surgery (MOPiS) is a multi-component intervention that addresses ${ }^{1}$ opioid overprescribing, for example by promoting baseline assessment of a patient prior to writing any opioid prescription to identify and address duplicative opioid prescriptions (eg, provider use of state prescription monitoring programmes (PMP) $)^{2} 344748$, optimising pain control (eg, use of non-opioid analgesics as first-line therapy with opioid supplementation, if needed $)^{49} 50$ and $^{3}$ minimising reliance on opioid prescriptions for pain control (eg, familiarisation of providers with evidence on alternatives to opioid analgesics and with the actual number of pills used by the patients for pain management). ${ }^{495152}$

\section{Study aims}

The MOPiS study was developed, based on current evidence and national guidelines, to address four distinct domains $^{1}$ : expectation setting ${ }^{2}$, baseline assessment ${ }^{3}$, optimisation of perioperative non-opioid pain management and $^{4}$ opioid minimisation at discharge.$^{51-54}$

We will conduct a mixed-methods evaluation of the implementation and effectiveness of MOPiS by ${ }^{1}$ applying a concurrent, formative evaluation to identify barriers and enablers to implementation ${ }^{2}$, using the Consolidated Framework for Implementation Research (CFIR) ${ }^{3}$, performing a quantitative assessment of site-specific effectiveness $a^{4} d^{4}$ determining the necessary and sufficient conditions for improving post-surgical opioid prescribing without negatively affecting pain-related patient outcomes.

\section{The study aims are}

Aim 1: evaluate the implementation of a multi-component intervention to reduce post-surgical opioid prescribing, while ensuring optimal pain control across six hospitals

Hypothesis 1: Implementation requires site-specific contextual adaptations of the intervention by speciality-specific, multidisciplinary surgical teams.

Aim 2: assess changes in opioid prescribing and pain-related patient outcomes following implementation of the contextually specific, multi-component intervention

Hypothesis 2: Implementation of the intervention will lead to decreased post-surgical opioid prescriptions without a decline in patient-reported pain-related outcomes, compared with historical and contemporaneous controls.

Aim 3: assess the interaction between site-specific contextual factors and variation in the fidelity and intensity of implementation on post-surgical opioid discharge prescribing

Hypothesis 3: The context of the intervention, as well as, specific approaches to implementing the components of the intervention, will lead to greater improvement.

\section{Theoretical frameworks}

Implementation of the MOPiS intervention will be guided by the Exploration, Preparation, Implementation, Sustainment (EPIS) Framework. ${ }^{56}$ Figure 1 shows the adaptation of the EPIS Framework for MOPiS. In addition, the CFIR $^{55}$ will be used to comprehensively explore the contextual needs and adaptations of the intervention in order to achieve robust and sustainable implementation at each site.

\section{Study significance}

The MOPiS study will provide a generalisable framework for tailored implementation of post-surgical prescribing best practices to reduce patient and societal harm due 


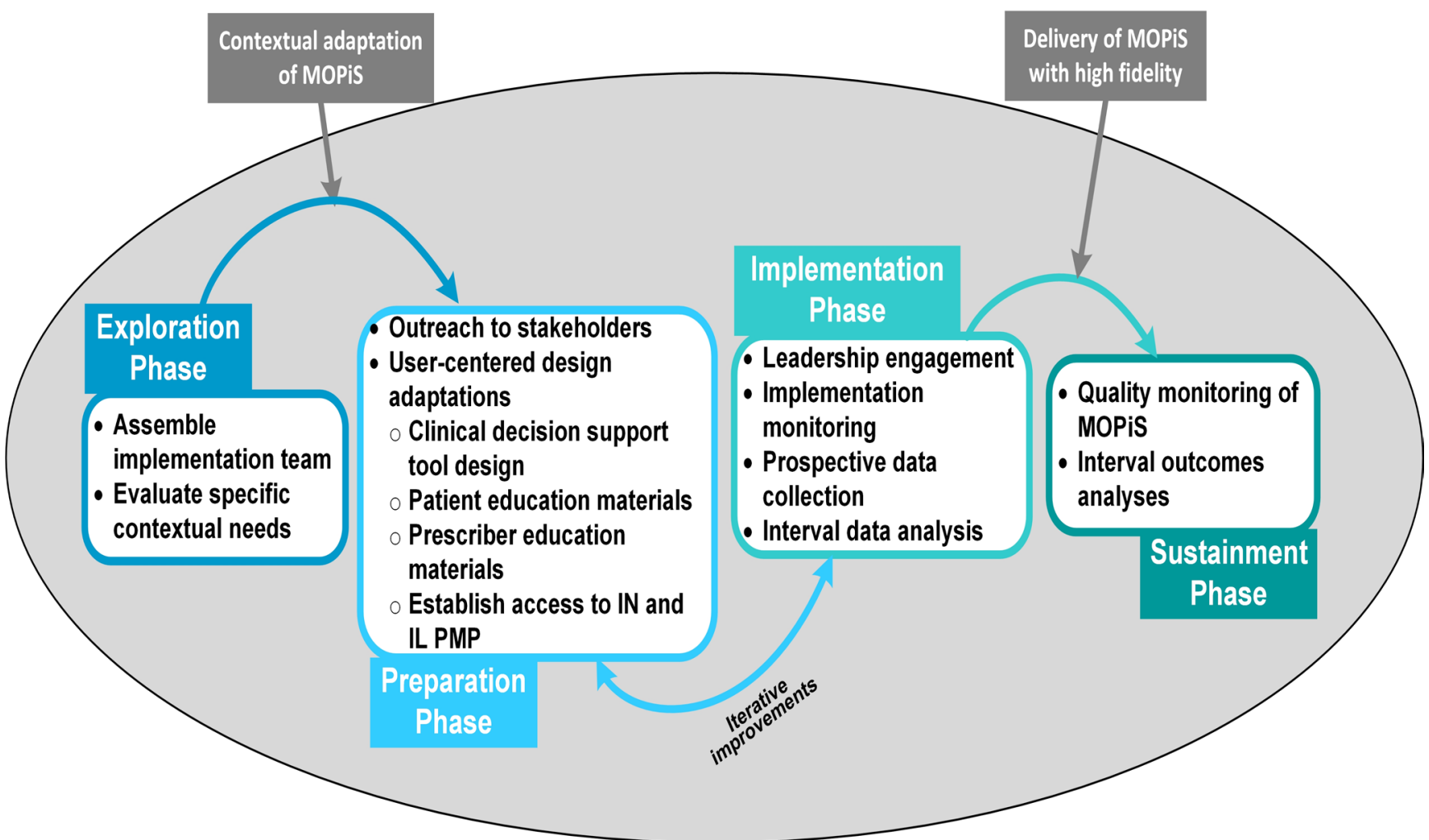

Figure 1 Application of EPIS phases to MOPiS Implementation. EPIS, Exploration, Preparation, Implementation, Sustainment; IN, Indiana; IL, Illinois; MOPiS, Minimising Opioid Prescribing in Surgery; PMP, prescription monitoring programmes.

to excess opioids without negatively affecting pain-related patient-reported outcomes. The study promotes evidence-based practices to identify effective strategies for contextually specific, system-level change, and it will provide both conceptual and practical insights to inform implementation of a system-level, evidence-based practice intervention in diverse hospitals, across the USA.

\section{METHODS AND ANALYSIS}

\section{Overview of study design}

The intervention will be implemented in four surgical areas (general, orthopaedics, urology and gynaecology) at six hospitals within a single health system. We will conduct a mixed-methods evaluation using formative evaluation techniques including qualitative (eg, focus groups, interviews, observations) and quantitative (eg, process and outcome measure performance reports) data collection to identify barriers and facilitators to the implementation and effectiveness of the intervention. ${ }^{57}$

Data collection, analysis and interpretation will be continuous before, during and after implementation with identified barriers being fed back iteratively to the hospitals for site-specific contextual adaptations. In addition, we will perform a quantitative evaluation to determine the effectiveness of the intervention to decrease post-surgical discharge opioid prescribing without negatively affecting pain-related patient-reported outcomes. To determine effectiveness, we will also use cases from non-intervention surgical specialities (plastic surgery, vascular surgery and neurosurgery) as contemporaneous within-site controls. Once effective strategies are identified, they will be implemented in the non-intervention specialities for study equipoise. Finally, we will combine the qualitative and quantitative results to ascertain which components of the intervention, in combination with site-specific contextual factors, are most effective in reducing opioid prescribing. The goal is to identify the most effective hospital-type and speciality-specific intervention bundles.

\section{Setting}

The study will be conducted at six hospitals within a health system, in Illinois. The hospitals are diverse with significant differences in size, location, population served and level of academical or community focus (table 1) and have a single enterprise data warehouse (EDW) for data collection.

\section{Intervention}

For each of the four domains, the intervention includes one or more main components:

Patient expectation setting

- Standardised Patient Education: Patient education materials will be developed with the aim of improving adherence to safe opioid use. ${ }^{29} 3451525458$ Educational materials will include printed brochures, as well as, interactive patient modules to educate patients on how to safely use, store and dispose of opioids. All educational material will be user-informed and 
Table 1 Hospitals and specialities participating in MOPiS

\begin{tabular}{lllllll} 
& Hospital 1 & Hospital 2 & Hospital 3 & Hospital 4 & Hospital 5 & Hospital 6 \\
\hline Category & Large academic & $\begin{array}{l}\text { Large } \\
\text { community }\end{array}$ & $\begin{array}{l}\text { Large } \\
\text { community }\end{array}$ & $\begin{array}{l}\text { Small } \\
\text { community }\end{array}$ & $\begin{array}{l}\text { Small } \\
\text { community }\end{array}$ & $\begin{array}{l}\text { Small } \\
\text { community }\end{array}$ \\
Teaching status & Teaching & Non-teaching & Non-teaching & Non-teaching & Non-teaching & Non-teaching \\
No of beds & 894 & 198 & 392 & 159 & 98 & 25 \\
\hline
\end{tabular}

MOPiS, Minimising Opioid Prescribing in Surgery.

literacy-level testing will be performed by our patient advisory council.

Baseline assessment of opioid use

- Automated IL-PMP Lookup: A key approach to reducing the number of opioids available for diversion is to ensure that the patients cannot access multiple opioid prescriptions simultaneously. The Prescription Monitoring Programme (PMP) is an electronic database that tracks the prescribing and dispensing of controlled substances (Schedule II-V), including opioids, with data on individual patients and individual prescribers. ${ }^{59}$ Clinicians can $\log$ into the PMP to view a patient's prior filled prescriptions. As part of the intervention, the PMP database will be integrated into the electronic health record (EHR) at each hospital to allow for an automated connection and to overcome technical and nuisance barriers associated with manual PMP lookup. Moreover, each time a provider attempts to order opioids, we plan to design an automatic lookup in the Illinois PMP (IL-PMP), so that prior opioid prescriptions will automatically appear next to the new opioid order.

Perioperative pain control optimisation

- Online Provider Education: Interactive, web-based, educational modules will be developed to educate providers on safe opioid prescribing, leveraging multimodal pain control strategies, setting pain management expectations with the patients and educating the patients on opioid safety. Content experts in perioperative pain management will help to develop the modules. The modules will be targeted toward all surgical care providers, including surgeons, advanced practice providers, nurses and pharmacists.

Postoperative opioid minimisation

- Electronic Health Record-based CDS Tools: Computerised decision support (CDS) tools can change prescribing behaviour, prevent medical errors and improve evidence-based clinical practice. ${ }^{60-63}$ We will gather procedure-specific opioid use data and combine this with existing evidence for procedure-specific prescribing guidelines to develop guidelines for appropriate prescription opioid quantity by procedure. Discharge order sets will incorporate the recommended quantity as a default. Surgical providers will retain the option of increasing the quantity of opioid pills by providing a brief explanation. Additionally, the discharge order sets will also include pain management order sets with non-opioid pain management alternatives (eg, ibuprofen, ice packs).

- Monthly Comparative Prescribing Reports: We will develop automated monthly comparative prescribing reports for individual surgical providers, detailing their prescribing data compared with blinded peer data and compared with prescribing recommendations by surgical procedure.

Adaptation of intervention components

We anticipate variation in the extent to which each hospital and each speciality adapts the intervention components, depending on hospital and speciality-specific contextual factors. The formative evaluation of MOPiS, conducted throughout the study timeframe, will enable the identification and documentation of hospitaland speciality-specific adaptations and improvements to the components to meet hospital- and speciality-specific contextual needs.

\section{Characteristics of study participants}

To study the systems and processes of care involved in post-surgical pain medication prescribing, including opioid prescribing, surgical providers and staff participating in the implementation will be research study participants. Surgical providers and staff, as participants in the qualitative data collection, will be identified using purposive sampling, based on subject matter expertise. Additionally, the patients will be interviewed. Potential patient participants will be identified by the surgical providers. English-speaking adults of any gender and $\geq 18$ years of age who are willing to speak about their experience with the implementation of preoperative opioid education and expectation setting and post-surgical pain management will be invited to participate. Patients will be interviewed until saturation of themes is reached.

In addition, quantitative data about the patients who have undergone selected surgical procedures at each of the participating hospitals will be gathered for both intervention and control specialities, using a survey and electronic data abstraction from the EDW.

\section{Patient and public involvement}

To ensure that patient education materials are patient and family-centred, the health system's patient advisory council will review materials. Additionally, advisory panel members, including a patient safety foundation 
representative, will provide feedback on study materials and findings, and will support dissemination efforts.

\section{Data collection components}

- Readiness Assessment: Prior to implementation, we will conduct a survey to assess the readiness of each hospital and each speciality to implement the necessary process changes and to identify additional needed resources. The survey will be conducted among all surgical providers involved in the implementation of the intervention.

- Interviews: We will conduct semi-structured confidential interviews with key stakeholders at each hospital and within each speciality to include, but not be limited to, clinic nurses, post-anaesthesia care unit (PACU) nurses, surgeons, advanced practice nurses, physician assistants, pharmacists and surgical residents (where applicable). Interview questions will focus on assessing the local adaptation and implementation of each intervention component, as well as, the individual's perceptions of the utility of each component. We will specifically inquire about barriers to change, including questions about the culture and work environment, in order to gain input about any site-specific barriers and facilitators during implementation.

- Focus Groups: During the implementation phase, we will conduct focus groups at each hospital, using a set of structured questions to guide the discussion. Focus group participants will include an attending physician, a PACU nurse, an outpatient clinic nurse, a resident or physician assistant where applicable and a representative from each speciality's administrative leadership. Each focus group will consist of six to eight participants and last for approximately 1 hour. The data from the focus groups will provide important information about issues related to the division's participation in the intervention.

- Observations: We will conduct a series of ethnographical observations of preoperative clinical encounters at each hospital to help us understand hospital and speciality-specific contextual factors that affect the adaptation and implementation of the preoperative components (eg, observe the functioning of a preoperative clinic and patient encounter) ${ }^{64-66}$ The observer will assume the role of 'peripheral-member researcher,' which brings an 'outsider's perspective' to the observation to allow for accurate appraisal of activities. The site visit team will collect data using a semi-structured observation tool. ${ }^{67-69}$ We will use observation to assess the patient exposure to the intervention components.

- Patient Interviews: We will conduct interviews with a random selection of patients in each hospital to ask the patients about their experiences with, and exposure to, opioid education, expectation setting and post-surgical pain management to provide real-time patient satisfaction and exposure data to the implementation team.
- Medical Chart Abstraction: Using data abstraction queries from the system-wide enterprise data warehouse (EDW), we will develop an automated report to identify prescribing patterns and process measure adherence by domain of optimal use, as detailed in table 2. In addition, the EDW will generate information on hospital characteristics and contextual factors. To validate the EDW data and any data that cannot be queried through the EDW, certified data abstractors will conduct manual chart reviews on a random sample of eligible patient charts.

- Patient Surveys: We will use online surveys to collect pain-related patient-reported outcomes among the patients who have undergone selected surgical procedures at each of the participating hospitals within the intervention and control specialities. Patients will be asked to rate their pain (0 to 10) daily for the first 7 days following discharge and, the morning of each day (postoperative day 2 to 8 ), they will be asked to report the number of opioid pills consumed in the prior 24hours. The Patient-Reported Outcomes Measurement Information System (PROMIS) Pain Intensity Short Form will be administered using computerised adaptive testing preoperatively, and then at 2 weeks, 3 months, 6 months and 1 year post-surgically.

Table 3 provides a summary of the different data collection components.

- Secondary Data Sources: Data on hospital characteristics will be obtained from the American Hospital Association Annual Hospital Survey and the Centres for Medicare and Medicaid Services Payment Update Impact Files, including: bed size, discharge volume, surgical case volume, resident-to-bed ratio/teaching status, ownership, case mix index, core-based statistical area and nurse-to-bed ratio.

\section{Data analyses}

Measures of intervention effectiveness

- The primary outcome will be the number of pills prescribed per surgical case (patient/case-level measure).

- Secondary outcomes include the strength, quantity and formulation of each post-surgical opioid prescribed, converted to morphine milligram equivalents.

- The electronical survey will use PROMIS Pain Intensity Short Form questions that capture how pain interferes with a patient's quality of life over the preceding 7 days. This will function as a balancing measure to assess potential negative impacts of the intervention.

\section{Quantitative data analysis}

We will perform a quantitative evaluation to determine the effectiveness of the intervention to decrease post-surgical discharge opioid prescribing without negatively affecting patient-reported pain outcomes.

- Change in Opioid Prescribing: A comparison of the mean number of pills prescribed per case post-intervention 
Table 2 Process measures for optimal postoperative opioid use

\begin{tabular}{|c|c|c|c|c|c|}
\hline Setting & Domain & Process measure & Measure & $\begin{array}{l}\text { Implementation } \\
\text { outcomes }\end{array}$ & Variable type \\
\hline \multirow[t]{5}{*}{ Preoperative } & \multirow[t]{2}{*}{ Expectation setting } & \multirow[t]{2}{*}{$\begin{array}{l}\text { Preoperative narcotic } \\
\text { education }\end{array}$} & $\begin{array}{l}\text { Preoperative education } \\
\text { documented in preop } \\
\text { note or preop clinic note }\end{array}$ & Fidelity & dichotomous \\
\hline & & & $\begin{array}{l}\text { Opioid education tool } \\
\text { distributed }\end{array}$ & Fidelity & dichotomous \\
\hline & \multirow[t]{3}{*}{ Baseline assessment } & $\begin{array}{l}\text { Chronic opioid use } \\
\text { investigated }\end{array}$ & $\begin{array}{l}\text { PMP user look-up } \\
\text { registry }\end{array}$ & Fidelity & dichotomous \\
\hline & & \multirow[t]{2}{*}{$\begin{array}{l}\text { Chronic pain } \\
\text { addressed }\end{array}$} & $\begin{array}{l}\text { Chronic pain tool } \\
\text { distributed }\end{array}$ & Fidelity & dichotomous \\
\hline & & & $\begin{array}{l}\text { Referral to pain } \\
\text { specialist }\end{array}$ & Fidelity & dichotomous \\
\hline \multirow[t]{5}{*}{ Perioperative } & \multirow{5}{*}{$\begin{array}{l}\text { Optimising pain } \\
\text { control (minimising } \\
\text { opioid use) }\end{array}$} & $\begin{array}{l}\text { Preoperative } \\
\text { analgesic given }\end{array}$ & $\begin{array}{l}\text { EMR MAR from OR } \\
\text { or anaesthesia record }\end{array}$ & Exposure & dichotomous \\
\hline & & $\begin{array}{l}\text { Pre-incision local } \\
\text { anaesthetic }\end{array}$ & $\begin{array}{l}\text { Dictated in operative } \\
\text { note }\end{array}$ & Exposure & dichotomous \\
\hline & & Anaesthesia type & Anaesthesia Record & Exposure & categorical \\
\hline & & $\begin{array}{l}\text { Anaesthesia adjuncts } \\
\text { (eg, regional block, } \\
\text { epidural, intravenous } \\
\text { lidocaine, etc) }\end{array}$ & Anaesthesia record & Exposure & categorical \\
\hline & & $\begin{array}{l}\text { Multimodal pain } \\
\text { control }\end{array}$ & $\begin{array}{l}\text { EMR MAR from inpatient } \\
\text { stay }\end{array}$ & Exposure & dichotomous \\
\hline \multirow{2}{*}{ Postoperative } & \multirow{2}{*}{ Opioid minimisation } & \multirow[t]{2}{*}{$\begin{array}{l}\text { Discharge education } \\
\text { information provided }\end{array}$} & $\begin{array}{l}\text { Post-surgical pain } \\
\text { handout provided }\end{array}$ & Fidelity & dichotomous \\
\hline & & & $\begin{array}{l}\text { Documentation of } \\
\text { education in discharge } \\
\text { record }\end{array}$ & Fidelity & dichotomous \\
\hline
\end{tabular}

EMR, electronic medical record; MAR, medication administration record; NIDA, National Institute on Drug Abuse; OR, operating room; PCP, primary care physician; PMP, prescription monitoring programmes.

(Years 2 and 3) compared with pre-intervention (Year 1) will be performed, clustered within surgeons, specialities and hospital. Because the intervention is at the system-level and the cluster size may not support three or four levels of clustering, analyses will be designed to account for the 24 clusters at the site-speciality level (table 1). First, we will assess pre- and post-intervention differences in mean number of pills prescribed per case, using a one-sided paired Student's t-test with clustered SE (or Wilcoxon rank-sum test for non-normally distributed data) to test the hypothesis that our intervention will reduce the mean number of pills prescribed per case. We will also use statistical models with controls for patient, speciality and hospital characteristics. We will explore several models to assess appropriateness and robustness of findings across specifications. Approaches may include a linear ordinary least squares fixed effects regression model of the form:

$$
Y_{i j t}=\alpha+\beta_{1} \text { Year } 2_{2}+\beta_{2} \text { Year3 }+\theta X_{i j t}+\gamma \text { Specialty }_{\text {Site }}+u_{i j t}
$$

where $Y_{i j t}$ is the number of pills prescribed for each case $i$, in speciality-site $j$, in year $t ; \alpha$ is an intercept, $\beta_{1}$ and $\beta_{2}$ are coefficients on dummy variables indicating cases from the post-intervention period, $X_{i j t}$ is a vector of patient covariates and SpecialtySite $j$ is a set of 23 speciality-site 
Table 3 Summary of data collection components and measures

\begin{tabular}{|c|c|c|c|c|}
\hline \multirow{2}{*}{$\begin{array}{l}\text { Data collection } \\
\text { component }\end{array}$} & \multicolumn{3}{|c|}{ Implementation phase (EPIS framework) } & \multirow[b]{2}{*}{ Measures } \\
\hline & Exploration & Preparation & Implementation Sustainment & \\
\hline $\begin{array}{l}\text { Readiness } \\
\text { assessment }\end{array}$ & & & & Readiness to implement, resources needed \\
\hline Observations & & & & $\begin{array}{l}\text { Patient exposure to education and } \\
\text { expectation setting }\end{array}$ \\
\hline Focus groups & & & & $\begin{array}{l}\text { Issues related to division's participation in } \\
\text { intervention }\end{array}$ \\
\hline Patient interviews & & & & $\begin{array}{l}\text { Patient exposure to education and } \\
\text { expectation setting, patient satisfaction }\end{array}$ \\
\hline $\begin{array}{l}\text { Secondary data } \\
\text { sources }\end{array}$ & & & & Hospital characteristics \\
\hline
\end{tabular}

EPIS, Exploration, Preparation, Implementation, Sustainment.

indicators ("fixed effects") which control for all time-invariant speciality-site characteristics, measured and unmeasured, that only vary across speciality-sites but are invariant within. In effect, this conservatively controls for all between-speciality-site differences and identifies treatment differences within sites. We hypothesise that the intervention will reduce the number of prescribed pills per case, thus we anticipate $\beta_{1}<0$ and $\beta_{2}<0$. A more stringent test of sustained improvement over time implies that $\beta_{2} \& l t ; \beta_{1} \& l t ; 0$. Because fixed effects linear models preclude the inclusion of any speciality-site covariates that may be of interest, we will also explore estimation of two-level hierarchical linear models with speciality-site random intercepts.

- Intervention Effect on Case-Level Patient-Reported Outcomes Measurement Information System Pain Intensity Short Form Pain Scores: Pre-post and difference-in-differences, as outlined above, will be calculated. However, we hypothesise that the coefficients on study year will not be statistically different from zero.

- Sensitivity Analyses: We will do several sensitivity analyses, including ${ }^{1}$ : with and without inclusion of patient and speciality covariates in the models ${ }^{2}$; examining the association between hospital, surgical speciality, procedural characteristics and missing data and $^{3}$ examining spillover effects by assessing operations within the hospital similar to those studied but performed by surgeons outside of the targeted specialities, serving as contemporaneous controls (eg, emergency general surgery or acute care surgery procedures).

- Power Calculations: The sample size is expected to be stable in the four specialities in each hospital (table 1). On average, we expect approximately 1888 cases per speciality by site. We conducted a baseline assessment of opioid prescribing and found a mean of 30 pills and SD of 10. Assuming a baseline prescription rate of 30 pills per case, a conservative hypothetical estimate of speciality by site intra-cluster correlations of 0.2 , alpha $=0.05$ and power $=80 \%$, we anticipate being able to detect a minimal difference of 4.58 pills in mean rates before-and-after the intervention if the SD remains 10 . This would increase to a minimum detectable decrease of 9.16 pills assuming $\mathrm{SD}=20$, or minimal detectable difference of 16.21 pills if the $\mathrm{SD}=50$.

\section{Measures of implementation effectiveness}

- Feasibility: Conducting pre-implementation interviews with key stakeholders at each hospital will provide important information about perceived barriers to the intervention and whether interviewees think it will be possible to implement the intervention.

- Fidelity: Throughout the implementation, we will use data from the EDW queries to measure the extent to which each component is actually implemented, using the process measures noted above. Fidelity of implementation will also be assessed through the preoperative clinic observations.

- Exposure: The degree to which the intervention components were actually experienced by the patient will be measured through data abstracted from the EDW, observations of preoperative clinic encounters and patient surveys. 


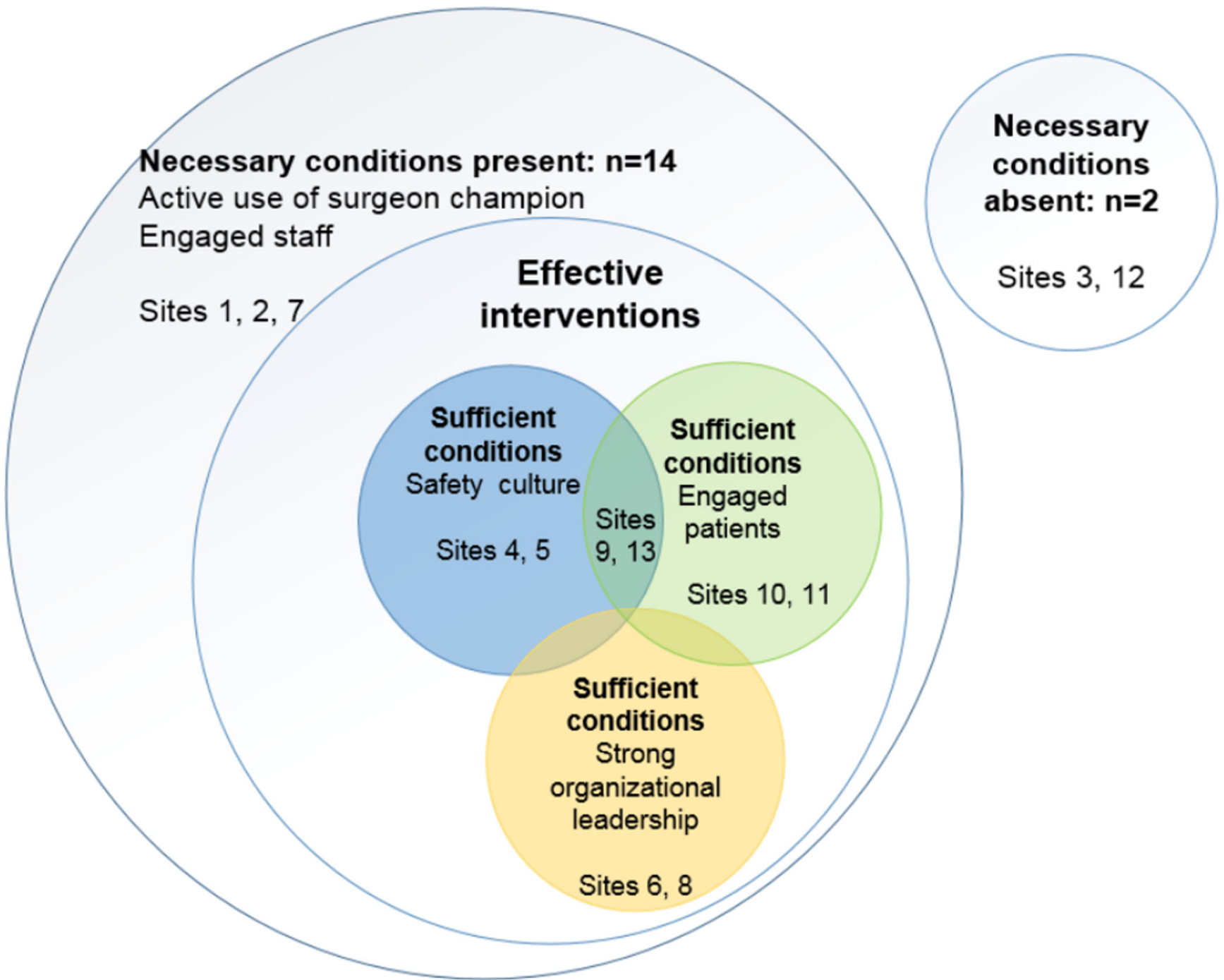

Figure 2 Example of present and absent conditions for outcomes to occur.

- Intensity: We will estimate a summary score, based on clinician and patient surveys, observations and focus groups.

\section{Qualitative comparative analysis}

In the final phase of the study, we will assess which components of the intervention, in combination with site-specific contextual factors, are most effective in reducing opioid prescribing. Specifically, we will conduct Qualitative Comparative Analysis (QCA) by combining the results of the qualitative and quantitative data to identify the necessary and sufficient conditions for improved opioid prescribing while preventing negative pain-related patient outcomes.

Using QCA, we will be able to identify more than one causal pathway to the outcome of interest (eg, reducing opioid prescribing) and identify conjunctional causation or the conditions that may only display their effects in conjunction with other conditions. QCA is a case-oriented mathematical approach that examines relationships between conditions and an outcome. ${ }^{70}$ QCA answers the question: what conditions, alone or in combination with other conditions, are necessary or sufficient to produce the outcome of interest?

- Data: The first step in QCA is to develop a conceptual model, based on input from the qualitative results. The transcripts from the surveys, interviews and observations will be coded and analysed to identify potential causal relationships. The research team will use a computer-assisted qualitative data analysis software (MAXQDA) to support the analysis. During analysis, categorical themes will be identified and applied to further transcripts. We will conduct a hybrid form of textual analysis which combines both inductive and deductive logics. ${ }^{71} 72$ The analytical strategy will be informed by the task at hand (assessment of the implementation of the intervention), as well as, the desire to allow unanticipated themes to emerge from the data and to allow participant understandings to be revealed, as in our prior work. ${ }^{73} 74$ Themes identified from the qualitative research will provide insight into site-specific implementation adaptations and how participants anticipate implementation of 
the intervention to reduce opioid prescribing while effectively controlling post-surgical pain. Results of the analysis will be used to build the QCA conceptual model. The conceptual model will emphasise the conditions that need to be in place, either individually or in combination, for the intervention to be effective at a specific hospital (ie, the necessary conditions) and what conditions, either individually or in combination, would produce the outcome in a specific hospital (ie, sufficient conditions). For example, using the results of the qualitative research, we might find the conditions most likely to influence implementation success include use of a surgeon champion, engaged staff, safety culture, engaged patients and strong organisational leadership. Figure 2 illustrates a hypothetical example that considers the necessary and sufficient set-theoretical relationships. As shown in the figure, necessary conditions must be present for an outcome to occur.

- Analysis: Crisp-set QCA analysis will be used to dichotomise the conditions for each hospital as either 'having' or 'not having' each condition. Data from the 24 sites within the six hospitals will be rank-ordered from highest to lowest on two outcomes (opioid prescriptions and pain-related patient measures). A 'truth table' will be constructed to assess whether all logically possible configurations have empirical cases. At the conclusion of the truth table analysis, we will return to the qualitative data to provide rich examples of the cases.

- Sensitivity Analyses: Sensitivity analyses will examine relationships between change scores and numerous formulations of the domain-specific implementation scores to assess the robustness of the findings.

\section{ETHICS AND DISSEMINATION}

In this study, we describe the protocol for implementation and evaluation of a multifaceted opioid minimisation intervention. The study aims to identify the most effective hospital-type and speciality-specific intervention bundles for rapid dissemination into our 56-hospital learning collaborative and in hospitals throughout the USA. Dissemination activities will be further supported by our External Advisory Panel which includes public health leaders at the federal, state and city levels, as well as experts in medication diversion and addiction, public safety and health and drug policy and patient advocacy. Furthermore, findings will be disseminated through a combination of traditional approaches (peer-reviewed publications and conference presentations) and newer technology-driven approaches (social media accounts, websites and webinars). Dissemination materials will be prepared as soon as data are available and when analyses have been finalised.

\section{Author affiliations}

${ }^{1}$ Surgical Outcomes \& Quality Improvement Centre (SOQIC), Department of Surgery, Northwestern University Feinberg School of Medicine, Northwestern University, Chicago, Illinois, USA
${ }^{2}$ Centre for Healthcare Studies, Institute for Public Health and Medicine, Northwestern University Feinberg School of Medicine, Northwestern University, Chicago, Illinois, USA

${ }^{3}$ Centre for Communication and Health, Institute for Public Health and Medicine, Northwestern University Feinberg School of Medicine, Northwestern University, Chicago, Illinois, USA

Contributors JJS conceptualised the study, developed the quantitative analysis plan and wrote significant portions of the manuscript. JJ also conceptualised the study, developed the qualitative analysis plan and wrote significant portions of the manuscript. WLAS, RH and MLS supported in writing and editing of the manuscript and detailing the study methodology. BL, JLH and KYB supported in the study conceptualisation and critically reviewed various drafts of the manuscript. All authors read and approved the final manuscript.

Funding Research reported in this publication was supported by the National Institute on Drug Abuse of the National Institutes of Health under Award Number R34DA044752. Funding for this study runs from 1 September, 2017, through 31 August, 2020. The content is solely the responsibility of the authors and does not necessarily represent the official views of the National Institutes of Health.

Competing interests None declared.

Patient consent for publication Not required.

Ethics approval All study activities have been approved by the Northwestern Universitylnstitutional Review Board (ID STU00205053).

Provenance and peer review Not commissioned; peer reviewed for ethical and funding approval prior to submission.

Open access This is an open access article distributed in accordance with the Creative Commons Attribution Non Commercial (CC BY-NC 4.0) license, which permits others to distribute, remix, adapt, build upon this work non-commercially, and license their derivative works on different terms, provided the original work is properly cited, appropriate credit is given, any changes made indicated, and the use is non-commercial. See: http://creativecommons.org/licenses/by-nc/4.0/.

\section{REFERENCES}

1. Stulberg JJ BE, Nooromid MJ. Analysis of Northwestern Medicine's Electronic Data Warehouse, 2016.

2. Ringwalt C, Gugelmann H, Garrettson M, et al. Differential prescribing of opioid analgesics according to physician specialty for Medicaid patients with chronic noncancer pain diagnoses. Pain Res Manag 2014;19:179-85.

3. Bates C, Laciak R, Southwick A, et al. Overprescription of postoperative narcotics: a look at postoperative pain medication delivery, consumption and disposal in urological practice. J Urol 2011;185:551-5.

4. Morris BJ, Mir HR. The opioid epidemic: impact on orthopaedic surgery. J Am Acad Orthop Surg 2015;23:267-71.

5. Hill MV, McMahon ML, Stucke RS, et al. Wide Variation and Excessive Dosage of Opioid Prescriptions for Common General Surgical Procedures. Ann Surg 2017;265:709-14.

6. Gray J, Hagemeier N, Brooks B, et al. Prescription Disposal Practices: A 2-Year Ecological Study of Drug Drop Box Donations in Appalachia. Am J Public Health 2015;105:e89-e94.

7. Hasak JM, Roth Bettlach CL, Santosa KB, et al. Empowering PostSurgical Patients to Improve Opioid Disposal: A Before and After Quality Improvement Study. J Am Coll Surg 2018;226:235-40.

8. Lewis ET, Cucciare MA, Trafton JA. What do patients do with unused opioid medications? Clin J Pain 2014;30:654-62.

9. Levy B, Paulozzi L, Mack KA, et al. Trends in Opioid AnalgesicPrescribing Rates by Specialty, U.S., 2007-2012. Am J Prev Med 2015;49:409-13.

10. Substance Abuse and Mental Health Services Administration. Key Substance Use and Mental Health Indicators in the United States: Results from the 2016 National Survey on Drug Use and Health. (HHS Publication No. SMA 17-5044, NSDUH Series H-52). Rockville, MD: Center for Behavioral Health Statistics and Quality, Substance Abuse and Mental Health Services Administration, 2017.

11. Jones CM, Paulozzi LJ, Mack KA. Sources of prescription opioid pain relievers by frequency of past-year nonmedical use United States, 2008-2011. JAMA Intern Med 2014;174:802-3.

12. Centers for Diseases Control and Prevention (CDC). 2018 Annual Surveillance Report of Drug-Related Risks and Outcomes - United States: CDC National Center for Injury Prevention and Control, 2018. 
13. Wide-ranging online data for epidemiologic research (WONDER. GA: Atlanta: Statistics CNCfH, ed, 2017.

14. Centers for Disease Control and Prevention. Annual Surveillance Report of Drug-Related Risks and Outcomes - United States, 2017. Surveillance Special Report 1: Centers for Disease Control and Prevention, U.S. Department of Health and Human Services, 2017.

15. Centers for Diseases Control and Prevention (CDC). U.S. Opioid Prescribing Rate Maps 2018. 2018 https://www.cdc.gov/ drugoverdose/maps/rxrate-maps.html (Updated 3 Oct 2018)

16. Guy GP, Zhang K. Opioid Prescribing by Specialty and Volume in the U.S. Am J Prev Med 2018;55:e153-e155.

17. Volkow ND, McLellan TA, Cotto JH, et al. Characteristics of opioid prescriptions in 2009. JAMA 2011;305:1299-301.

18. Alam A, Gomes $\mathrm{T}$, Zheng $\mathrm{H}$, et al. Long-term analgesic use after low-risk surgery: a retrospective cohort study. Arch Intern Med 2012;172:425-30.

19. Bartels K, Mayes LM, Dingmann C, et al. Opioid use and storage patterns by patients after hospital discharge following surgery. PLoS One 2016;11:e0147972.

20. Breivik H. How to implement an acute pain service. Best Pract Res Clin Anaesthesiol 2002;16:527-47.

21. Calcaterra SL, Yamashita TE, Min SJ, et al. Opioid Prescribing at Hospital Discharge Contributes to Chronic Opioid Use. J Gen Intern Med 2016;31:478-85.

22. Carroll IR, Hah JM, Barelka PL, et al. Pain duration and resolution following surgery: an inception cohort study. Pain Med 2015;16:2386-96.

23. Carroll I, Barelka P, Wang CK, et al. A pilot cohort study of the determinants of longitudinal opioid use after surgery. Anesth Analg 2012:115:1-702.

24. Clarke HA, Katz J, McCartney CJ, et al. Perioperative gabapentin reduces $24 \mathrm{~h}$ opioid consumption and improves in-hospital rehabilitation but not post-discharge outcomes after total knee arthroplasty with peripheral nerve block. Br J Anaesth 2014;113:855-64.

25. Clarke $\mathrm{H}$, Poon $\mathrm{M}$, Weinrib $\mathrm{A}$, et al. Preventive analgesia and novel strategies for the prevention of chronic post-surgical pain. Drugs 2015;75:339-51.

26. Clarke H, Soneji N, Ko DT, et al. Rates and risk factors for prolonged opioid use after major surgery: population based cohort study. BMJ 2014;348:g1251.

27. Dowell D, Zhang K, Noonan RK, et al. Mandatory Provider Review And Pain Clinic Laws Reduce The Amounts Of Opioids Prescribed And Overdose Death Rates. Health Aff 2016;35:1876-83.

28. Kennedy-Hendricks A, Gielen A, McDonald E, et al. Medication Sharing, Storage, and Disposal Practices for Opioid Medications Among US Adults. JAMA Intern Med 2016;176:1027-9.

29. Dualé $C$. Prolonged use of opioids after surgery. $B M J$ 2014;348:g1280.

30. Gomes T, Juurlink DN, Dhalla IA, et al. Trends in opioid use and dosing among socio-economically disadvantaged patients. Open Med 2011;5:e13-22.

31. Greer SM, Dalton JA, Carlson J, et al. Surgical patients' fear of addiction to pain medication: the effect of an educational program for clinicians. Clin J Pain 2001;17:157-64.

32. Huang A, Katz J, Clarke H. Ensuring safe prescribing of controlled substances for pain following surgery by developing a transitional pain service. Pain Manag 2015;5:97-105.

33. Chaparro LE, Smith SA, Moore RA, et al. Pharmacotherapy for the prevention of chronic pain after surgery in adults. Cochrane Database Syst Rev 2013;7:CD008307.

34. Society ISM. Recommendations for Deterring Improper Use of Opioids, 2015

35. Mason MJ, Golladay G, Jiranek W, et al. Depression Moderates the Relationship Between Pain and the Nonmedical Use of Opioid Medication Among Adult Outpatients. J Addict Med 2016;10:408-13.

36. McDonald DC, Carlson K, Izrael D. Geographic variation in opioid prescribing in the U.S. J Pain 2012;13:988-96.

37. Menendez ME, Mellema JJ, Ring D. Attitudes and self-reported practices of hand surgeons regarding prescription opioid use. Hand 2015;10:789-95.

38. Mixter CG, Meeker LD, Gavin TJ. Preemptive pain control in patients having laparoscopic hernia repair: a comparison of ketorolac and ibuprofen. Arch Surg 1998;133:432-7.

39. Gordon DB, Dahl J, Phillips P, et al. The use of "as-needed" range orders for opioid analgesics in the management of acute pain: a consensus statement of the American Society for Pain Management Nursing and the American Pain Society. Pain Manag Nurs 2004;5:53-8.
40. Rodgers J, Cunningham K, Fitzgerald K, et al. Opioid consumption following outpatient upper extremity surgery. J Hand Surg Am 2012;37:645-50.

41. Sun EC, Darnall BD, Baker LC, et al. Incidence of and Risk Factors for Chronic Opioid Use Among Opioid-Naive Patients in the Postoperative Period. JAMA Intern Med 2016;176:1286-93.

42. Katz J, Weinrib A, Fashler SR, et al. The Toronto General Hospital Transitional Pain Service: development and implementation of a multidisciplinary program to prevent chronic postsurgical pain. J Pain Res 2015;8:695-702.

43. Hahn KL. Strategies to prevent opioid misuse, abuse, and diversion that may also reduce the associated costs. Am Health Drug Benefits 2011:4:107-14

44. Burcu M, Zito JM, Metcalfe L, et al. Trends in Stimulant Medication Use in Commercially Insured Youths and Adults, 2010-2014. JAMA Psychiatry 2016;73:992-3.

45. Zhou C, Florence CS, Dowell D. Payments For Opioids Shifted Substantially To Public And Private Insurers While Consumer Spending Declined, 1999-2012. Health Aff 2016;35:824-31.

46. Bao Y, Pan Y, Taylor A, et al. Prescription Drug Monitoring Programs Are Associated With Sustained Reductions In Opioid Prescribing By Physicians. Health Aff 2016;35:1045-51.

47. Services DoHaH. Prescription Drug Monitoring Program Interoperability Standards: A Report to Congress. Washington, DC 2013.

48. Finklea KSL, Bagalman E. Prescription Drug Monitoring Programs. Congressional Research Service CRS Report Prepared for Members and Committees of Congress 2014.

49. Chou R, Gordon DB, de Leon-Casasola OA, et al. Management of Postoperative Pain: A Clinical Practice Guideline From the American Pain Society, the American Society of Regional Anesthesia and Pain Medicine, and the American Society of Anesthesiologists' Committee on Regional Anesthesia, Executive Committee, and Administrative Council. J Pain 2016:17:131-57.

50. Renthal W. Seeking Balance Between Pain Relief and Safety: CDC Issues New Opioid-Prescribing Guidelines. JAMA Neurol 2016;73:513-4.

51. Dowell D, Haegerich TM, Chou R. CDC Guideline for prescribing opioids for chronic pain - United States, 2016. MMWR Recomm Rep 2016;65:1-49.

52. American Society of Anesthesiologists Task Force on Acute Pain Management. Practice guidelines for acute pain management in the perioperative setting: an updated report by the American Society of Anesthesiologists Task Force on Acute Pain Management. Anesthesiology 2012;116:248-73.

53. Council NS. Prescription Nation 2016, 2016.

54. Administration SAaMHS. SAMHSA Opioid overdose prevention toolkit. 2016. Report No: (SMA) 16-4742.

55. Damschroder LJ, Aron DC, Keith RE, et al. Fostering implementation of health services research findings into practice: a consolidated framework for advancing implementation science. Implement Sci 2009;4:50.

56. Aarons GA, Hurlburt M, Horwitz SM. Advancing a conceptual model of evidence-based practice implementation in public service sectors. Adm Policy Ment Health 2011;38:4-23.

57. Creswell JW. A concise introduction to mixed methods research: SAGE publications, 2015

58. Michie S, Johnston M, Abraham C, et al. Making psychological theory useful for implementing evidence based practice: a consensus approach. Qual Saf Health Care 2005;14:26-33.

59. Yeh B. Legal Issues Relating to the Disposal of Dispensed Controlled Substances, 2010.

60. Slight SP, Berner ES, Galanter W, et al. Metadata Correction: Meaningful Use of Electronic Health Records: Experiences From the Field and Future Opportunities. JMIR Med Inform 2015;3:e32.

61. Galanter WL, Thambi M, Rosencranz H, et al. Effects of clinical decision support on venous thromboembolism risk assessment, prophylaxis, and prevention at a university teaching hospital. Am J Health Syst Pharm 2010;67:1265-73.

62. Galanter W, Falck S, Burns M, et al. Indication-based prescribing prevents wrong-patient medication errors in computerized provider order entry (CPOE). J Am Med Inform Assoc 2013;20:477-81.

63. Galanter WL, Bryson ML, Falck S, et al. Indication alerts intercept drug name confusion errors during computerized entry of medication orders. PLoS One 2014;9:e101977.

64. Benning A, Ghaleb M, Suokas A, et al. Large scale organisational intervention to improve patient safety in four UK hospitals: mixed method evaluation. BMJ 2011;342:d195.

65. Reeves S, Kuper A, Hodges BD. Qualitative research methodologies: ethnography. BMJ 2008;337:a1020. 
66. Taxis K, Barber N. Ethnographic study of incidence and severity of intravenous drug errors. BMJ 2003;326:684.

67. Barach P, Johnson JK, Ahmad A, et al. A prospective observational study of human factors, adverse events, and patient outcomes in surgery for pediatric cardiac disease. J Thorac Cardiovasc Surg 2008;136:1422-8.

68. Bognár A, Barach P, Johnson JK, et al. Errors and the burden of errors: attitudes, perceptions, and the culture of safety in pediatric cardiac surgical teams. Ann Thorac Surg 2008;85:1374-81.

69. Johnson JK, Barach P, Vernooij-Dassen M, et al. Conducting a multicentre and multinational qualitative study on patient transitions. BMJ Qual Saf 2012;21 Suppl 1(Suppl 1):i22-i28.

70. Ragin C. The comparative method: Moving beyond qualitative and quantitative strategies. Berkeley: University of California Press, 1987:85-118.
71. Lofland J, Lofland L. Analyzing social settings. Belmont, CA: Wadsworth Publishing Company, 2006.

72. Miles M, Huberman A, Saldana J. Qualitative Data Analysis: A Methods Sourcebook2014. Los Angeles, CA: SAGE Publications Inc, 2014.

73. Johnson JK, Woods DM, Stevens DP, et al. Joy and challenges in improving chronic illness care: capturing daily experiences of academic primary care teams. J Gen Intern Med 2010;25 Suppl 4(Suppl 4):581-5.

74. Stevens DP, Bowen JL, Johnson JK, et al. A multi-institutional quality improvement initiative to transform education for chronic illness care in resident continuity practices. J Gen Intern Med 2010;25 Suppl 4:574-80. 